\title{
Combining Top-down and Bottom-up Visual Saliency for Firearms Localization
}

\author{
Edoardo Ardizzone, Roberto Gallea, Marco La Cascia and Giuseppe Mazzola \\ DICGIM, Universita' degli Studi di Palermo, Palermo, Italy \\ \{edoardo.ardizzone, roberto.gallea, marco.lascia, giuseppe.mazzola\}@unipa.it
}

\begin{abstract}
Keywords: Firearms Detection, Visual Saliency, Probabilistic Model.
Abstract: Object detection is one of the most challenging issues for computer vision researchers. The analysis of the human visual attention mechanisms can help automatic inspection systems, in order to discard useless information and improving performances and efficiency. In this paper we proposed our attention based method to estimate firearms position in images of people holding firearms. Both top-down and bottom-up mechanisms are involved in our system. The bottom-up analysis is based on a state-of-the-art approach. The top-down analysis is based on the construction of a probabilistic model of the firearms position with respect to the people's face position. This model has been created by analyzing information from of a public available database of movie frames representing actors holding firearms.
\end{abstract}

\section{INTRODUCTION}

The human visual system is able to easily detect an interesting object in natural scenes through the selective attention mechanism, that discard useless information, selecting the most relevant ones for higher-level cognitive processing. The attention process selects visual information on the basis of both saliency in the image (bottom-up, task-independent process), and of prior knowledge about the context and the objects in the scene (top-down, task dependent process). In the top-down process, attention detects salient areas through understanding and recognition mechanisms. Bottom-up processing is a primitive function of the human vision system and responds to various stimuli such as intensity, color, and orientation, etc. In a generic scene analysis, both of them are integrated for a faster visual search. Models of integration are natural in human vision, but are difficult to define for computer vision applications. In fact, searching for a particular object in a scene can be extremely difficult, as one has to consider all possible views that the object can take. In our work we are interested in searching for firearms locations, particularly in scenes where people are holding firearms. The proposed system is based on a combination of a bottom-up saliency map and top-down information, obtained from the analysis of the relative positions of the firearm with respect to the face of a person in the scene. In the next section we illustrate some state-of-the-art methods for visual saliency analysis and attention based object recognition. In section 3 we present our firearms localization system. In section 4 we discuss and evaluate our experimental results. A conclusive section ends the paper.

\section{PREVIOUS WORKS}

Many computational implementations of visual attention models have been published in the past years. Several works proposed algorithms to extract bottomup saliency information, exploiting different features: multiscale information (Itti et al., 1998), graph based activation maps (Harel et al., 2007), colors (Liu et al., 2011; Kovacs and Sziranyi, 2007), isophotes and color histograms (Valenti et al., 2009), distribution of the interest points (Ardizzone et al., 2011). The authors of (Judd et al., 2009) studied the relationship between computer generated saliency maps and maps created by tracking the gaze of people looking at a number of test images. Other works focused on topdown information: (Gao et al., 2009), (Kanan et al., 2009), (Oliva et al., 2003). In literature there are also some theories on mechanisms of integration between bottom-up and top-down information: Feature Integration Theory (Treisman and Sato, 1990), Biased Competition Model (Desimone and Duncan, ), Guided Search (Wolfe, 1994), Optimal Gains (Navalpakkam and Itti, 2007). Many scientific works rely on 
object recognition, which often focus on two aspects of the problem: extracting features from images, and classifying these features.

Even if Computer Vision researchers achieved impressive results on object detection in the last years (Lowe, 2004; Viola and Jones, 2004), this is still an open research field. Many factors, such as changes in viewpoint and scale, illumination, partial occlusions and multiple instances further complicate the problem of object detection (Uijlings et al., 2013; Felzenszwalb et al., 2010; Vedaldi et al., 2009; Lopez et al., 2012). Attentional frameworks have been proposed to speed up the visual search (Bonaiuto and Itti, 2005) without exploiting top-down knowledge about the target. The VOCUS-model from (Frintrop, 2006) use both a bottom-up and a top-down version of the saliency map: the bottom-up map is similar to that of Itti and Koch's, while the top-down map is a tuned version of the bottom-up one, and the total saliency map is a linear combination of the two maps with user provided weights. The authors of (Oliva et al., 2003) show that top-down information extracted from the context of the scene can modulate the saliency of image regions during the task of object detection. Regarding firearms detection, that is the topic of our paper, notwithstanding the importance of the topic in the era of social network and anti-terrorism struggles for the authorities, just a few works were proposed. Among these (Zhang and Blum, 1997; Yang and Blum, 2002; Xue and Blum, 2003) proposed techniques to reveal concealed firearms, by fusing information from multiple sources (thermal/infrared (IR), millimeter wave (MMW), and visual sensors). However, to the best of our knowledge, no methods based solely on image information exist.

\section{PROPOSED SYSTEM}

The proposed system is based on the combination of the information from two different attention processes: a bottom-up saliency map and a top-down saliency map. Figure 1 shows the scheme of the overall system. Regarding the bottom-up analysis, we used in our system the GBVS approach by (Harel et al., 2007) which is based on a biologically plausible model, and it consists of two steps: activation maps on certain feature channels and normalization, which highlights conspicuity. The top-down analysis is based on the construction of a probabilistic model, able to estimate the regions of an image where a firearm is more likely to be found, with respect to the position of the person's face. The main idea is to build the statistics of a large set of samples and then fit a model onto it, which is then applied to every image to analyze. This approach will be further explained in the next subsections, after the description of dataset used to create the probabilistic model.

\subsection{Dataset Description}

Due to the large number of images required, a comprehensive dataset had to be acquired. However, a large realistic database with a variety of firearms is hard to be built from scratch. For this reason the images available in the "Internet Movie Firearms Database" (IMFDB) ${ }^{1}$ were used. The database is composed of several thousands images taken from movie scenes. Each image represents one or more persons holding one or more firearms. Images are middle quality color spanning from 0.06 to 2 megapixels. Figure 2 shows some examples of images taken from the database.

\subsection{Dataset Annotation}

In order to obtain reliable statistics from the images, they required to be manually annotated with some labels. Then, several metrics were measured. In particular, 1000 images were labeled with the following information:

- Image filename;

- Image size, both horizontal $I_{w}$ and vertical $I_{h}$;

- Firearm position $W_{p x}$ and $W_{p y}$ and size, both horizontal $W_{w}$ and vertical $W_{h}$.

- Face position $F_{p x}$ and $F_{p y}$ and size, both horizontal $F_{w}$, and vertical $F_{h}$.

From these elements, additional information is extracted, namely:

- Distance from face to firearm $d_{w-f}$ normalized w.r.t. face size;

- Orientation of the firearm w.r.t. the subject face $\alpha_{w-f}$;

- The area of the firearm bounding box;

- The area of the face bounding box.

Note that each measure is normalized w.r.t. face sizes, in order to make the values comparable notwithstanding the subject size or image resolution.

\footnotetext{
${ }^{1}$ Internet Movie Firearms Database (IMFDB) http://www.imfdb.org/
} 


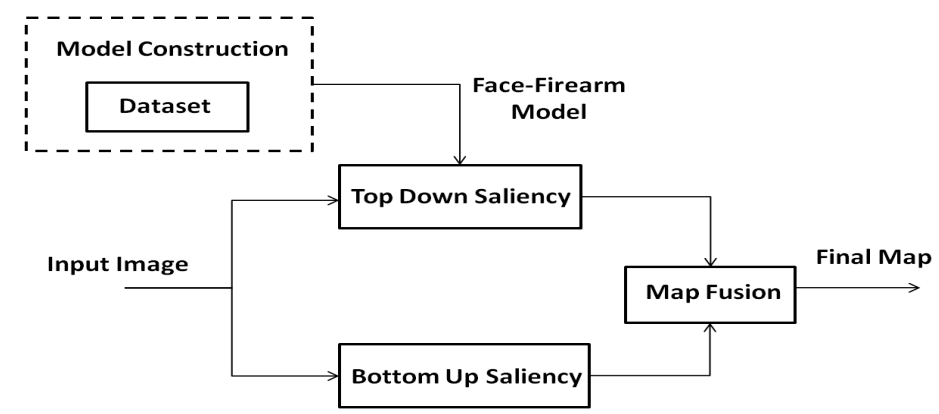

Figure 1: Blocks diagram for the proposed system.

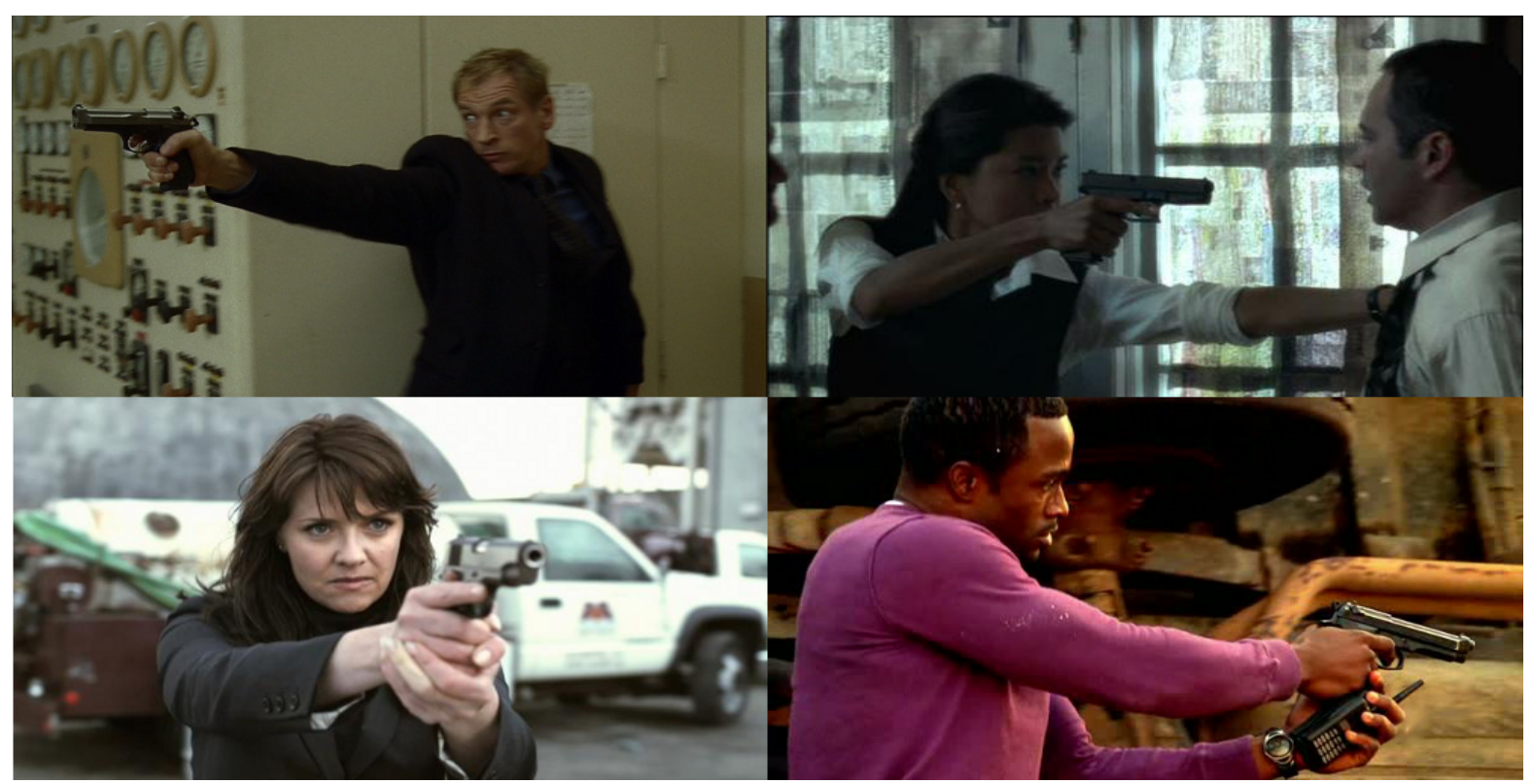

Figure 2: Example images from the Internet Movie Firearms Database - IMFDB.

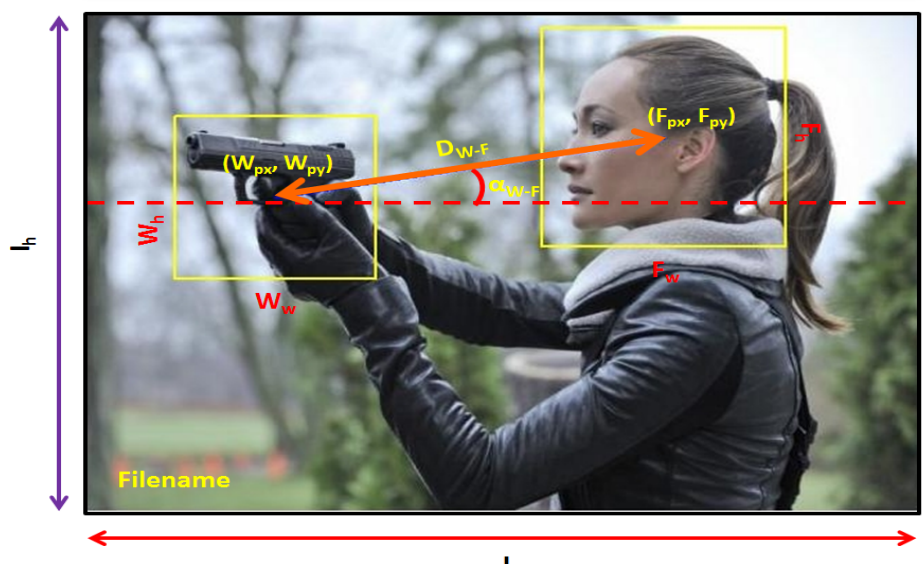

$I_{w}$

Figure 3: Annotated measures for the dataset: Horizontal $I_{w}$ and vertical $I_{h}$ image size; firearm position $W_{p x}$ and $W_{p y}$ and size, both horizontal $W_{w}$ and vertical $W_{h}$; face position $F_{p x}$ and $F_{p y}$ and size, both horizontal $F_{w}$, and vertical $F_{h}$.

\subsection{Model Construction}

The key idea is that a person holding a firearm has a pose which generally follows some constraints, due to physical reasons (for example, his/her arm extension), or more practical ones (for example he/she is taking aim), making the probability distribution of the firearm position not-uniform across the image. Some 


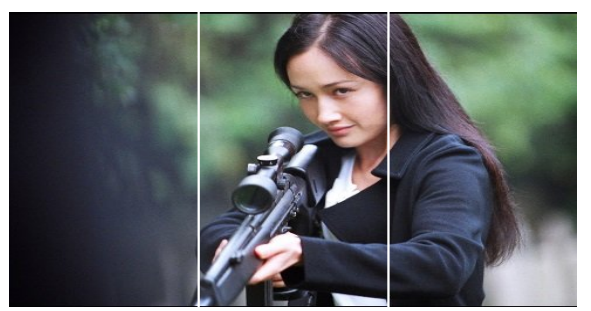

(a)

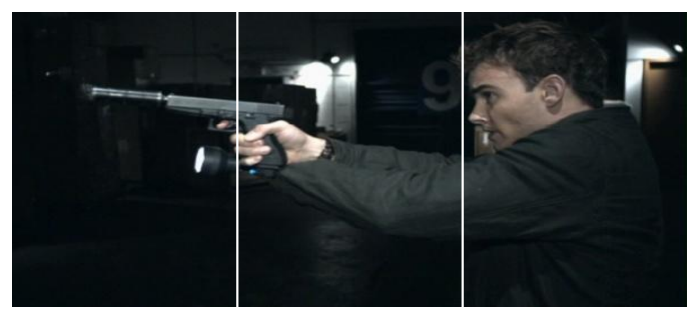

(b)

Figure 4: Two examples of image subdivision to categorize the images w.r.t. face position.

other considerations could be done. As an example, considering a subject head located on the left of the image, the firearm is very likely to lie on its right (and viceversa). Moreover, if the head is large w.r.t. the whole image, the firearm is likely to lie close to the face (the image is a portrait of an aiming person). Following these considerations, the whole dataset was subdivided according to two criteria: face position and face extensions w.r.t. image sizes. For the first categorization, each image was partitioned into three regions as shown in Figure 4. The purpose of using such regions is mainly to find out if the subject is on the left, center or right. Note that an horizontal subdivision was not required because faces are located only in the upper regions of the images. The second categorization is made on a face size basis. If the face surface is at least as large as the $30 \%$ of the whole image surface, the face is labeled as "large", otherwise as "small". These are only two possible types of models that can be built by analyzing the dataset information.

The Firearm Probability Map is computed as the a posteriori probability of the firearm position, conditioned by the face position, registered with respect to the face center and rescaled with respect to the face size: Eqs. $(1,2)$

$$
\begin{gathered}
P_{w}(x, y)=P\left(W_{p x}^{\prime}, W_{p y}^{\prime} \mid F_{p x}, F_{p y}\right), \\
W_{p x}^{\prime}=\frac{W_{p x}-F_{p x}}{F_{w}} W_{p y}^{\prime}=\frac{W_{p y}-F_{p y}}{F_{h}},
\end{gathered}
$$

where

- $\left(W_{p x}, W_{p y}\right)$ and $\left(F_{p x}, F_{p y}\right)$ are the coordinates of the center of mass of the bounding box that includes the firearm and the face, respectively, in the inspected images of the dataset;

- $\left(W_{p x}^{\prime}, W_{p y}^{\prime}\right)$ are the registered and rescaled coordinates of the weapons.

- $F_{w}$ and $F_{h}$ are the horizontal and the vertical size of the bounding box of the detected face.

For creating the required model, a statistics over some dataset quantities was assessed. In particular the joint histogram of $d_{w-f}$ and $\alpha_{w-f}$ was built. The result is shown in Figure 5 and Figure 6 respectively for face position and face size categorization. In the plot $x$ values refer to $d_{w-f}$ and $y$ values refer to $\alpha_{w-f}$. As expected, the distributions are considerably dissimilar in the different cases related both to face positions (Figure 5). Also, even when considering face size, is evident that when the face is large, the distance of the firearm from it is small and viceversa.

In the direction of building the probability maps, the joint histograms are converted from polar to cartesian coordinates, values are then low-passed to smooth the distribution. An example of the resulting maps is shown in Figure 7(b). Note that the coordinates of the map are normalized w.r.t. face size and are centered on $(x, y)=(0,0)$.

For applying the model to a test image, once the face position has been detected, the top-down map $M$ needs to be registered to the image by being traslated to the face center and scaled to reflect face size. Finally, the resulting map $M_{d}$ is recovered by combining $M$ with GBVS saliency $S_{G B V S}$. Such operation has a dual purpose:

- Filter out non-salient regions underlying the firearm probability map.

- Filter out salient regions NOT underlying the firearm probability map.

Scalar product between maps was chosen as integration operator (Eq.3).

$$
M_{d}=M \cdot S_{G B V S} .
$$

Figure 7(a-f) shows the whole process: the original image is used to guide the registration of the proper top-down probability map (according to face position and size). Then the map is integrated with the GBVS saliency to recover the final firearm saliency.

\section{EXPERIMENTAL TESTS, RESULTS AND DISCUSSION}

As described in Section 3, the top-down model has been built by analyzing 1000 images from the Internet Movie Firearms Database. In our tests we selected 


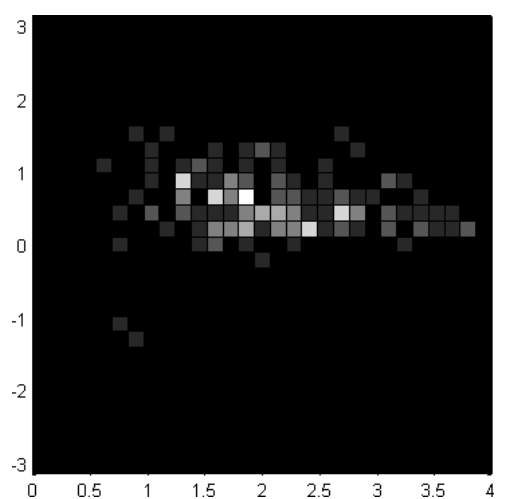

(a) Left

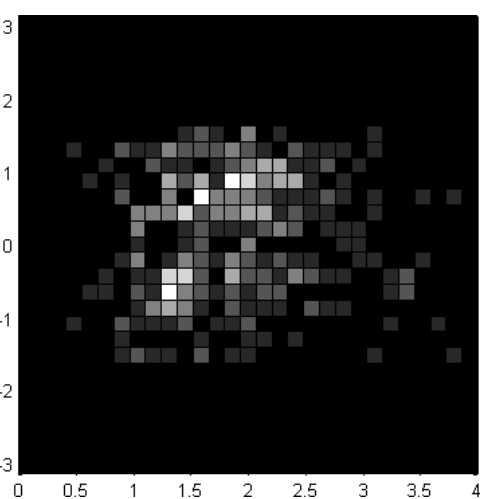

(b) Center

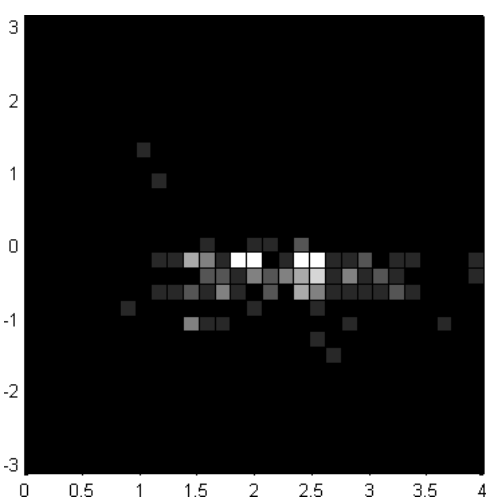

(c) Right

Figure 5: Joint histograms for $d_{w-f}$ and $\alpha_{w-f}$ for the three image categories related to face position. From (a) to (c) are shown histograms for faces located on the left (a), center (b) and right (c) regions of the images.

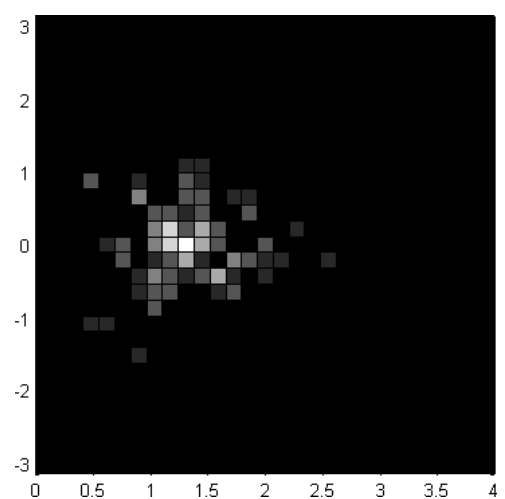

(a) Large

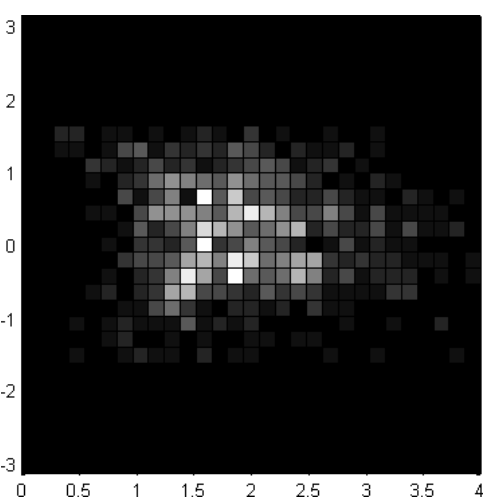

(b) Small

Figure 6: Joint histograms for $d_{w-f}$ and $\alpha_{w-f}$ for the two image categories related to face size, for large faces (a) and small faces (b) respectively. Note that when faces are large, distances are mostly small and vice-versa.

1000 more images from the database, and we annotated them by drawing the bounding box of the areas that include the firearms. Therefore, for each test image, we have a binary map that indicates the position of the firearm, that is our reference ground truth mask for that image. As well, the output map of the system is obtained from the input image, once the position of the face in the image is known. In this work we are not interested in the face detection process, as there are many works in literature (e.g. Viola and Jones (Viola and Jones, 2004)) that achieve impressive results. The output map is then thresholded, after normalization, by different threshold values, in the range of $[0,0.95]$ with step 0.05 . Finally, the bounding boxes of the connected components of the binarized map are taken as our output binary mask (see Figure 8 ). To evaluate the accuracy of our localization system we compare the binary mask of the detected map $M_{d}$, for a given threshold, with the reference binary mask $M_{r}$ of that image, in terms of recall, precision and F-measure:

$$
P=n \frac{\left(M_{d} \cap M_{r}\right)}{n\left(M_{d}\right)},
$$

$$
\begin{gathered}
P=\frac{n\left(M_{d} \cap M_{r}\right)}{n\left(M_{r}\right)}, \\
F_{1}=2 \frac{P \cdot R}{P+R}
\end{gathered}
$$

where:

- $R$ is the recall, the ratio of the number of pixels in the intersection of the detected mask $M_{d}$ and the reference mask $M_{r}$, and the number of pixels in $M_{r}$;

- $P$ is the precision, the ratio of the number of pixels in the intersection of the detected mask $M_{d}$ and the reference mask $M_{r}$, and the number of pixels in $M_{d}$;

- $F 1$ is the F-measure, that is the is the harmonic mean of precision and recall.

In our experiments we divided the test dataset into three subsets: images in which the faces are on the left, on the center or on the right part of the scene, then we evaluated three different top-down models. 


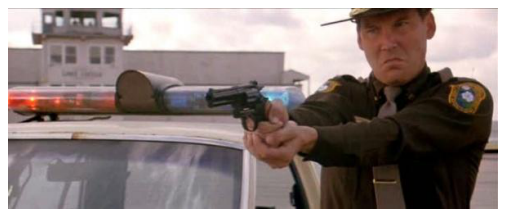

(a) Original image

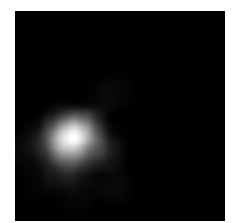

(b) top-down map

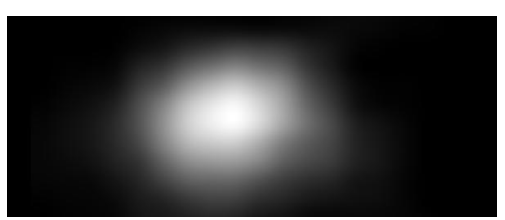

(c) Registered map

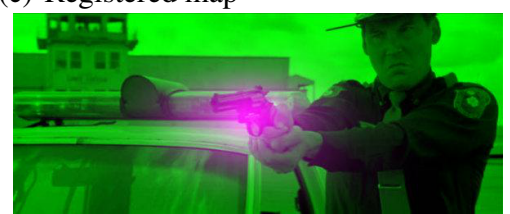

(f) Overlay

(d) GBVS map

(e) Integrated map

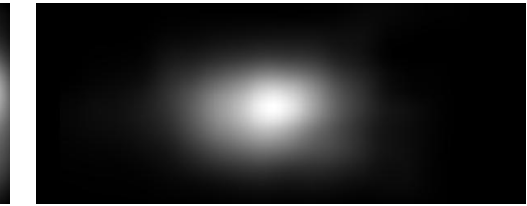

Figure 7: Firearm detection process: the original image (a) is used to determine which of the top-down top-down map (b) has to be used. The map is then registered onto the image, according to face position and size (c). GBVS saliency (d) is integrated with the top-down map to obtain the final firearm probability map (e). An overlay of the map with the original image is shown in (f).

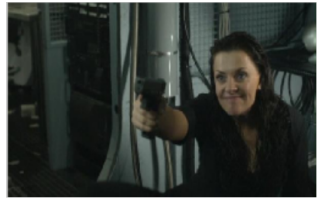

(a) Input image

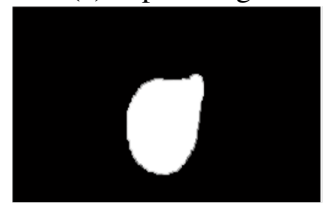

(c) Thresholded output mask

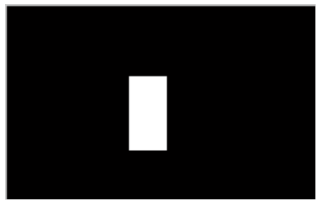

(b) Reference mask

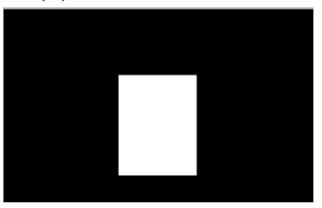

(d) Output bounding box

Figure 8: Evaluation process. Input image (a); reference mask, annotated by hand (b); binarized output mask (c) with threshold $=0.35$; bounding box (d) of the mask in (c). In our experiments we compare the mask in (b) with the mask in (d).

Figure 9(a-c) shows the results obtained in the three cases, and with all the data, in terms of recall, precision and F-measure. We observed that when the face is located on the right or on the left part of the image results are better than those obtained when the face is on the center. This could be reasonably expected as, when the person is on the center of the image, there is, more or less, the same probability to find a firearm on the left or on the right side of the person. In fact the top-down model, in this case, is symmetrical with respect of the face position, and the output masks include more pixels than in other two cases, resulting in a little bit higher recall and a much lower precision. The best threshold value is that which maximizes the F-measure, i.e. the best tradeoff between recall and precision, and it is equal to 0.35 for the "left" and "right" models, and to 0.4 for the "center" model. Results are very encouraging, especially for the "left" and the "right" models.

\section{CONCLUSIONS AND FUTURE WORKS}

In this paper we showed that top-down and bottomup information can be effectively integrated for object detection purposes. In particular in our work we are interested in finding the location of firearms in natural scenes representing people holding firearms. The algorithm would be extended to include firearm shape validation in order to suppress some false positives. We also consider to integrate information about the pose of the face in the firearm position estimate.

The proposed system has been designed to be a step of more complex architectures, with applications in video surveillance systems, crime prevention mechanisms, forensics analysis, etc. With this goal in our future works we plan to create a complete process pipeline for the analysis of images of armed persons: from people localization, to firearms position estima- 


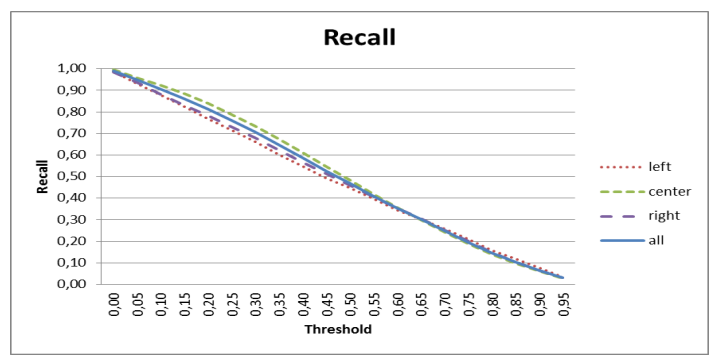

(a)

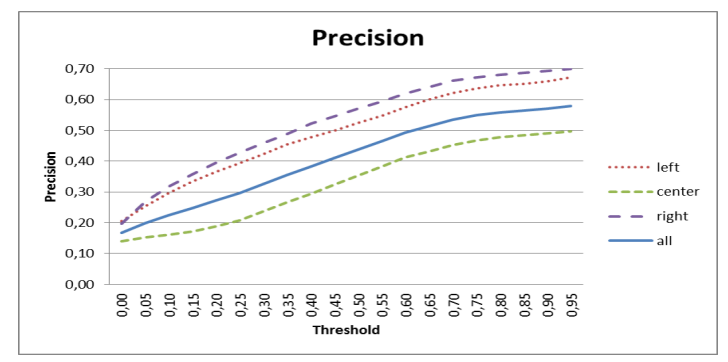

(b)

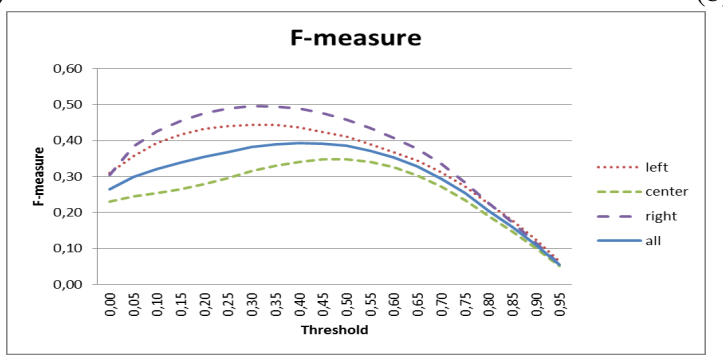

(c)

Figure 9: Experimental results: recall (a), precision (b), and F-measure (c). Results are shown for the three cases we analyzed in our experiments, in terms of the face position in the image (left, center and right), and with all the data.

tion (that is the topic of this paper), to firearm detection (to identify whether an object held in the hand by a person is a firearm or a harmless item) and classification (to distinguish between some firearm classes, once a firearm has been detected).

\section{ACKNOWLEDGEMENTS}

This work has been funded by the MIUR (Italian Ministry of Education, University and Research) Italian project PON01_01687, SINTESYS - Security and INTElligence SYStem. We also acknowledge Mr. Francesco Toto who contributed with his work to the implementation and in the testing phase.

\section{REFERENCES}

Ardizzone, E., Bruno, A., and Mazzola, G. (2011). Visual saliency by keypoints distribution analysis. In ICIAP (1), pages 691-699.

Bonaiuto, J. J. and Itti, L. (2005). Combining attention and recognition for rapid scene analysis. In Proceedings of the 2005 IEEE Computer Society Conference on Computer Vision and Pattern Recognition (CVPR'05) Workshops - Volume 03, CVPR '05, pages 90-, Washington, DC, USA. IEEE Computer Society.

Desimone, R. and Duncan, J. Neural mechanisms of selective visual attention. Annu Rev Neurosci, pages 193222.
Felzenszwalb, P., Girshick, R., McAllester, D., and Ramanan, D. (2010). Object detection with discriminatively trained part based models. Pattern Analysis and Machine Intellingence, 32(9).

Frintrop, S. (2006). VOCUS: A Visual Attention System for Object Detection and Goal-Directed Search. Lecture Notes in Artificial Intelligence. Springer.

Gao, D., Han, S., and Vasconcelos, N. (2009). Discriminant saliency, the detection of suspicious coincidences, and applications to visual recognition. IEEE Transactions on Pattern Analysis and Machine Intelligence, 31(6):989-1005.

Harel, J., Koch, C., and Perona, P. (2007). Graph-based visual saliency. In Advances in Neural Information Processing Systems 19, pages 545-552. MIT Press.

Itti, L., Koch, C., and Niebur, E. (1998). A model of saliency-based visual attention for rapid scene analysis. IEEE Transactions on Pattern Analysis and Machine Intelligence, 20(11):1254-1259.

Judd, T., Ehinger, K., Durand, F., and Torralba, A. (2009). Learning to predict where humans look. In IEEE International Conference on Computer Vision (ICCV).

Kanan, C., Tong, M. H., Zhang, L., and Cottrell, G. W. (2009). SUN: Top-down saliency using natural statistics. Visual cognition, 17(6-7):979-1003.

Kovacs, L. and Sziranyi, T. (2007). Focus area extraction by blind deconvolution for defining regions of interest. IEEE Trans. Pattern Anal. Mach. Intell., 29(6):10801085.

Liu, T., Yuan, Z., Sun, J., Wang, J., Zheng, N., Tang, X., and Shum, H.-Y. (2011). Learning to detect a salient object. IEEE Trans. Pattern Anal. Mach. Intell., 33(2):353-367.

Lopez, A. M., Vanrell, M., Bagdanov, A. D., Van de Wei- 
jer, J., Anwer, R. M., and Khan, F. S. (2012). Color attributes for object detection. 2013 IEEE Conference on Computer Vision and Pattern Recognition, 0:33063313.

Lowe, D. G. (2004). Distinctive image features from scaleinvariant keypoints. Int. J. Comput. Vision, 60(2):91110 .

Navalpakkam, V. and Itti, L. (2007). Search Goal Tunes Visual Features Optimally. Neuron, 53(4):605-617.

Oliva, A., Torralba, A., Castelhano, M. S., and M.Henderson, J. (2003). Top-down control of visual attention in object detection.

Treisman, A. and Sato, S. (1990). Conjunction search revisited. In Journal of Experimental Psychology: Human Perception and Performance, pages 459-478.

Uijlings, J. R. R., Van de Sande, K. E. A., Gevers, T., and Smeulders, A. W. M. (2013). Selective search for object recognition. International Journal of Computer Vision.

Valenti, R., Sebe, N., and Gevers, T. (2009). Image saliency by isocentric curvedness and color. In In ICCV.

Vedaldi, A., Gulshan, V., Varma, M., and Zisserman, A. (2009). Multiple kernels for object detection. In Proceedings of the International Conference on Computer Vision (ICCV).

Viola, P. and Jones, M. J. (2004). Robust real-time face detection. Int. J. Comput. Vision, 57(2):137-154.

Wolfe, J. M. (1994). Guided Search 2.0: A revised model of visual search. Psychonomic Bulletin \& Review, 1(2):202-238.

Xue, Z. and Blum, R. S. (2003). Concealed Weapon Detection Using Color Image Fusion. In Proceedings of 6th International Conference of Information Fusion, volume 1, pages 622-627.

Yang, J. and Blum, R. S. (2002). A statistical signal processing approach to image fusion for concealed weapon detection. In IEEE Intl. Conf. on Image Processing, pages 513-516. Marcel Dekker.

Zhang, Z. and Blum, R. S. (1997). Region-based image fusion scheme for concealed weapon detection. In In Proceedings of the 31st Annual Conference on Information Sciences and Systems, pages 168-173. 